\title{
Civilisations
}

Revue internationale d'anthropologie et de sciences

humaines

42-2 | 1993

enQuete d'identité

\section{Une cohabitation ethno-religieuse dans les Balkans : le cas du Rhodope oriental}

\section{Gueorguieva Tsvetana}

\section{OpenEdition \\ Journals}

Édition électronique

URL : http://journals.openedition.org/civilisations/2342

DOI : 10.4000/civilisations. 2342

ISSN : 2032-0442

\section{Éditeur}

Institut de sociologie de l'Université Libre de Bruxelles

\section{Édition imprimée}

Date de publication : 1 décembre 1993

Pagination : 161-174

ISBN : 2-87263-108-9

ISSN : 0009-8140

Référence électronique

Gueorguieva Tsvetana, « Une cohabitation ethno-religieuse dans les Balkans : le cas du Rhodope oriental », Civilisations [En ligne], 42-2 | 1993, mis en ligne le 01 décembre 1996, consulté le 30 avril 2019. URL : http://journals.openedition.org/civilisations/2342 ; DOI : 10.4000/civilisations.2342

Ce document a été généré automatiquement le 30 avril 2019

(c) Tous droits réservés 


\title{
Une cohabitation ethno-religieuse dans les Balkans : le cas du Rhodope oriental ${ }^{1}$
}

\author{
Gueorguieva Tsvetana
}

\section{Une zone de peuplement mixte chrétien et musulman}

1 Le Rhodope Oriental, comme beaucoup d'autres régions des Balkans, présente une mosaïque de populations d'origines, de confessions religieuses et de traditions culturelles différentes. La région est habitée depuis très longtemps par des Bulgares chrétiens et musulmans, par des Turcs sunnites et alevi (Kazilbas) et par des Tsiganes musulmans et chrétiens. C'est cette grande diversité ethnique et religieuse et les pratiques rituelles de la population autochtone qui ont motivé notre intérêt pour l'étude de la région.

\section{Les conditions d'enquête}

2 Les enquêtes ont été réalisées par 22 personnes : deux enseignants et vingt étudiants. Cinq villes (Kardzali, Krumovgrad, Momcilgrad, Dzebel et Ardino) ainsi que 26 villages ont été investigués ; 245 personnes appartenant à différentes communautés ethniques et religieuses y ont été interrogées².

3 La plupart des interviews dans les villages comme dans les villes se sont déroulées dans les maisons et à l'improviste, et dans des quartiers où vivent de façon compacte les populations mixtes, Ainsi par exemple à Kardzali, on a enquêté dans le "vieux hameau turc » où cohabitent déjà trois générations et dans le nouveau "complexe $»^{3}$ appelé «Vazrozdenci » (gens-de-la-renaissance), peuplé par des Musulmans et Chrétiens qui y cohabitent depuis peu. Il n'y a eu que deux cas où les enquêteurs ont été « delibérément manipulés »: une fois ce fut le maire du village de Kirkovo qui nous orienta vers des informateurs qui devaient prouver l'origine bulgare et la profonde conscience bulgare de la population locale; dans le deuxième cas, l'imam de Benkovski n'invita qu'une dizaine d'hommes dans la chambre de la Mosquée, limitant considérablement notre champ 
d'investigation. Celui-ci voulait prouver que les pratiques religieuses de la population sont essentiellement coraniques en dénigrant les variantes locales de culture populaire.

Il convient toutefois de souligner l'importance de ces deux rencontres "officielles» comme repère des actuelles tendances extrêmes de la société locale entre lesquelles s'insère toute une gamme de multiples tendances, divergentes, si ce n'est contradictoires.

Il faut noter ici que les enquêteurs n'ont rencontré aucun obstacle : quelle que soit leur religion et leur identité ethnique, tous les informateurs se sont prêtés avec plaisir à un entretien. Sans distinction d'âge, ni d'instruction, ils ont fait preuve de bonne volonté et personne n'a refusé de répondre. De toute évidence, la population locale qui, ces dix dernières années vit une situation perturbée, éprouve le besoin d'exprimer ses difficultés mais aussi ses opinions sur la situation passée et présente; elle recherche la compréhension et la reconnaissance de ses attitudes et de son expérience. A plus forte raison pour la deuxième série d'enquêtes qui a coïncidé avec d'intenses préparatifs électoraux pour l'Assemblée Nationale ordinaire, les informateurs, les hommes surtout, ont parlé de leur inquiétude et de leurs choix électoraux, sans manifester aucune hostilité à l'égard des enquêteurs. La grève des enfants turcs contre la décision de rendre facultative l'étude de leur langue maternelle, ne nous a pas non plus handicapée.

6 Sans doute y avait-il des tensions dans la région, mais elles ne se sont pas répercutées sur nos contacts avec les informateurs. Ceci atteste que le conflit y est moins violent qu'il n'y paraît sous sa forme politisée et même «mythologisée ».

\section{Le melting-pot rhodopéen : paysage historique et socioculturel}

\section{Les Pomak}

7 La formation de cette communauté multiethnique et multiconfessionnelle résulte d'une très longue évolution historique. La population bulgare installée ici depuis le Haut Moyen Age, habite la montagne. Une partie de ces montagnards bulgares furent islamisés au 15ème siècle, après la conquête du pays par la Dynastie Osmanlie. Ces Bulgares convertis, surnommés Pomak, sauvegardèrent leur langue maternelle et la plus grande partie de leurs traditions rituelles (Konstantinov et al., 1991). Les Pomak, à l'instar des Musulmans de Bosnie, représentent un groupement ethnique à conscience identitaire autonome qui joue un grand rôle médiateur dans l'interpénétration des civilisations chrétienne et musulmane des Balkans, mais qui subit des pressions considérables de la part des autres communautés voisines. Au 20ème siècle, au moment où les frontières étatiques des pays balkaniques ont été redessinées, une partie des Pomak se sont retrouvés dans les territoires de l'actuel État grec où ils sont aujourd'hui qualifiés d' "Hellènes musulmans slavophones" (Christakudis, 1992). Une masse assez importante de Pomak habite la Turquie où la presse les appelle « Turcs montagnards ».

Depuis la libération de la domination ottomane, les autorités bulgares, celles du gouvernement monarchiste, puis celles du régime totalitaire de la « République Populaire Démocratique " ont mené plusieurs campagnes musclées de bulgarisation portant atteinte à l'identité musulmane des Pomak. 
9 Après la guerre balkanique de 1912, en raison de l'installation de migrants venus des régions de la Thrace orientale rattachée depuis à la Turquie et à la Grèce, le nombre des Bulgares chrétiens dans la région commença à augmenter.

\section{Les Turcs}

L'essentiel de la population d'origine turque venue de l'Asie Mineure s'installe ici à partir des $15^{\text {ème }}-16^{\text {ème }}$ siècles - la grande époque de la colonisation ottomane des Balkans. Vraisemblablement, cette communauté a intégré une partie de la population autochtone. Toujours est-il qu'actuellement les Turcs rhodopiens sont musulmans, parlent turc et ont une conscience identitaire turque. Au cours des années 1984-85, ils avaient subi, comme tous les Turcs de Bulgarie, une grande pression assimilatrice : on a bulgarisé leurs noms et on leur a formellement interdit sous peine d'expulsion, d'emprisonnement et de tortures, de pratiquer leurs rites et de parler leur langue maternelle. Ces mesures oppressives sont à l'origine de la grande vague migratoire qui attribua à la Bulgarie l'image d'un État oppressif piétinant les droits élémentaires de l'homme.

\section{Les Têtes rouges}

11 La plupart des Turcs bulgares sont des sunnites, mais il existe un certain nombre d'Alevi shiites (Kazabas, littéralement "têtes rouges»), vraisemblablement originaires des territoires orientaux de l'Asie Mineure (Petkov et Fotev, 1990). Ils sont peu nombreux dans la région. Pour des raisons de conflits confessionnels, ils sont tenus à l'écart par les « vrais » musulmans (sunnites). Un Pomak âgé du village d'Egrek nous a expliqué qu'il ne fallait pas employer le mot kazilbas parce qu'il était injurieux (ACE, 1991). De même, un Turc d'Ardino disait que les Kazilbas sont «de mauvaises gens, pires que les Tsiganes »(ACE, 1991). Par ailleurs, les nombreux teke kazilbas de la région, notamment celui qui est situé près de Krumovgrad, sont fréquentés par tous les Musulmans rhodopiens. De même, les Kazilbas des villages mixtes (Raven, Tatul, Cobanka) observent strictement et sans entraves leurs rites shiites (ACE,1991). On pourrait dire donc que dans un grand nombre de cas la tolérance effective, manifestée par les musulmans sunnites dans la vie courante, infirme le rejet et les antagonismes théoriques.

\section{Les Tsiganes}

12 La présence de la population tsigane est très ancienne dans cette région, comme dans tous les pays balkaniques. Les Tsiganes sont bilingues: les Tsiganes musulmans parlent rom et turc, et les Tsiganes chrétiens parlent bulgare et rom. Indépendamment de leur appartenance religieuse, ils se considèrent eux-mêmes comme Tsiganes, et les autres les désignent comme tels également (Popov, 1992).

\section{L'habitat : aspects géographiques et démographiques}

Le relief de la région entre 200 et 600 mètres se caractérise par des gorges et de vastes vallées où sont situées en général les moyennes et petites villes (Krumovgrad, Momcilgrad, Kardzali) ; à côté de ces agglomérations urbaines, à même altitude, on trouve de nombreux villages. 

dispersa cette culture qui, par vagues successives, recouvrit les Balkans et l'Asie Mineure. La région du Rhodope oriental fait partie de la première zone de cette diffusion. Elle était proche de Constantinople, même du point de vue de la perception et de l'échelle médiévale des distances. Ce fonds commun fut à la fois suffisamment solide et souple pour tolérer les différentes innovations. L'influence croissante de la culture moderne et l'unification qu'elle entraîne conduisent à la formation d'une société industrielle aux nettes tendances cosmopolites. Au cours des siècles s'est produite une synthèse originale intégrant les différents courants culturels (Guéorguiéva, 1992).

\section{Des lunes et des saisons ${ }^{5}$}

L'une des caractéristiques des traditions du Rhodope oriental tient à l'adoption et à l'utilisation combinée de différents types de calendriers savants et empiriques, à savoir :

- a) le calendrier civil julien, puis sa version réajustée au l5ème siècle par le pape Grégoire XVIII. La version grégorienne de ce calendrier solaire savant introduit par Jules César en 46 av. J-C sous sa forme laïcisée, est devenu de nos jours le référent universel de toutes les notations calendaires existantes ${ }^{6}$;

- b) le calendrier religieux orthodoxe dont le férial christique (b1), essentiel du point de vue doctrinaire, est intégré dans un registre combiné du cycle lunaire et solaire. Le restant du férial, c'est-à-dire le cycle des fêtes patronales des saints (b2), s'inscrit entièrement dans le cycle saisonnier (solaire) ;

- c) le calendrier empirique des pastoraux nomades du désert froid qui est un calendrier dont la division saisonnière bipartite est ordonnée par le lever et le coucher héliaques des Pléiades ;

- d) le calendrier savant arabe, qui est un calendrier lunaire normalisé. Il ordonne le cycle de la liturgie islamique ; 
- e) le calendrier lunaire empirique, universel dans ses principes pour toutes les civilisations traditionnelles.

\section{Les fêtes canoniques} fluctuations annuelles limitées, est incompatible avec le férial représentatif islamique (Kurban bayram, Ramadan bayram, Kadir gedressi), qui est, lui, exclusivement lunaire et donc autonome par rapport à toute détermination saisonnière. Ainsi par exemple, la fête luni-solaire de Pâques ne coïncide pratiquement jamais avec aucun des bayrams liturgiques. Ces fêtes canoniques sont célébrées séparément par chacune des communautés religieuses. Toutefois les voisins «infidèles" d'en face ou d'à côté bénéficient toujours d'offrandes et de présents rituels. En effet, lors des bayrams, les musulmans offrent une partie du kurban’à leurs voisins chrétiens et en reçoivent, à la fête de Pâques, des oeufs peints en rouge. Au Ramadan bayram on offre aussi des mekici (beignets) et des bonbons aux enfants chrétiens du village. L'échange des cadeaux sacrés est accompagné obligatoirement de souhaits pour la fête respective, signe de l'estime mutuelle. appellent Paskale ${ }^{8}$. A cette occasion, tout comme les Bulgares, les Pomak confectionnent des oeufs peints.

Le jour de Noël (Koleda), qui est, avec l'Épiphanie l'une des rares fêtes christiques inscrites dans le registre des fêtes solaires (saisonnières), les villages étaient jadis parcourus par les koledari qui présentaient à chaque maison visitée des "louanges» (slavi)et des bénédictions (blagosloovi). Ces processions de jeunes chanteurs de «noëls » ne manquaient jamais de visiter également les maisons musulmanes. Cette coutume est de nos jours périmée, mais les informateurs pomak âgés se souviennent encore de l'accueil chaleureux que l'on réservait aux koledari (ACE, 1991).

作 religieuses hebdomadaires: les dimanches pour les Chrétiens et le vendredi pour les Musulmans. Ces jours-là, traditionnellement, on n'entre pas en conflit avec les gens de l'autre groupe. Ces dernières années le nombre des fidèles chez les chrétiens tout comme chez les musulmans a sensiblement augmenté, mais les deux groupes font preuve de respect mutuel. Cela ne veut pas dire pour autant que les vieilles rancunes sont complètement enterrées. En effet, si les jeunes musulmans et chrétiens de Kardzall s'entendent pour protester contre le chant du muezzin, leurs motivations sont profondément divergentes: pour les premiers il serait traité de coutume désuète introduite artificiellement, pour les seconds cet élément public du rituel religieux musulman serait interprété comme une marche triomphale.

\section{Les fêtes saisonnières}

Le calendrier civil (a), le férial orthodoxe des saints (b2) et le calendrier pastoral "pléïadique» sont tous les trois d'essence solaire et de ce fait leurs partitions saisonnières, inscrites dans le même cycle astronomique et bio-climatique, sont parfaitement compatibles. Ces partitions saisonnières, hormis leurs déterminations 
socioculturelles, sont évidemment tributaires des rythmes des travaux agricoles et pastoraux communs à tous les Rhodopiens ruraux. C'est à ce niveau de rites saisonniers que l'on rencontre la performance synchronisée et parfois fortement synchrétisée des fêtes célébrées par tous les Rhodopiens.

Les plus connues de ces manifestations sont la Saint-Georges du 23 avril et la SaintDimitri du 26 octobre du calendrier orthodoxe, correspondant respectivement au Hadrelez et au Kasim des Musulmans. Les fêtes de la Saint-Georges/Hadrelez et de la Saint-Dimitri/Kasim marquent le découpage de l'année du calendrier (c) populaire commun aux musulmans et aux chrétiens en deux saisons : estivale et hivernale. Sur le plan techno-économique, la première de ces dates correspond au moment de l'ouverture des pâturages montagnards, au sevrage des agneaux, et à la séparation des béliers des troupeaux des brebis; la seconde correspond à l'époque de l'accouplement des moutons et, dans le passé, au moment du départ pour les pâturages lointains. La popularité de ces fêtes s'explique entre autres par le fait que l'élevage des moutons fut longtemps pratiqué par tous les groupes rhodopiens.

Bien que les deux fêtes soient connues et respectées par tous, la plus grande notoriété revient à la Saint-Georges/Hadrelez solennellement célébrée par des rites communs à tous les groupes. Le jour de la fête, les gens se lèvent à l'aube, avant le lever du soleil pour aller se rouler dans la rosée et agiter des branches de clématite. Tôt matin on pratique aussi la divination au moyen de bouquets trempés dans des chaudrons déposés sous des rosiers; plus tard les jeunes gens accrochent aux arbres des balançoires et on les pousse en se balançant les uns les autres. Le point culminant de la fête est le sacrifice sanglant (kurban) de l'agneau que l'on consomme ensemble lors du festin collectif. La fête comporte une série de pratiques obligatoires et convergentes qui toutes marquent l'idée d'un commencement - depuis la confection rituelle du four portatif (podniea ), jusqu'à la première traite de lait de l'année. Ces pratiques rituelles sont communes aux Bulgares, aux Pomak et aux Turcs (ACE, 1991).

Selon l'un des informateurs du village Kajloba, on ne fête plus le Hadrelez parce qu'il coïncide avec la période la plus contraignante de la culture du tabac. Mais tous les autres informateurs turcs ont démenti cette interprétation. En fait, cette coïncidence entre les deux fêtes est utilisée comme argument pour prétendre que les Turcs de Bulgarie sont en fait d'origine bulgare.

La population musulmane exécute plusieurs de ces pratiques rituelles en leur attribuant des interprétations islamiques : le chaudron divinatoire serait consacré à la Mosquée, l'un des bouquets trempés serait dédié à Allah (ACE, 1991). Cette "islamisation » suggère le fonctionnement d'un mécanisme qui délimite, sur le plan confessionnel et ethnique, les éléments communs du rite, alors que le sens de la fête est général. On considère la SaintGeorges/Hadrelez comme fête de la joie, de la chance et du bonheur.

La population urbaine, détachée des rythmes naturels, a sensiblement modifié le rite en n'en sauvegardant que quelques éléments, tels l'ouverture obligatoire des fenêtres pour faire entrer les rayons de soleil, l'aération des vêtements en laine, le rôtissage d'un agneau dont la consommation sera abondamment arrosée de boissons alcoolisées. Ces pratiques fragmentaires sont communes aux Turcs, aux Bulgares et aux Pomak. Tout le monde affirme cependant que si le 23 avril tombe un jour férié, on va "à ciel ouvert " (ACE, 1991). 

en bulgare et sous l'appellation turque de mevlid présente un intérêt particulier. La variante chrétienne diffère considérablement par rapport à la performance rituelle musulmane. Il faut signaler cependant que les Pomak la pratiquent à la manière et sous l'appellation bulgare. Le jour convenu les villageois se réunissent pour verser de l'eau sur la tête d'une petite fille orpheline couverte de verdure qui fera ensuite le tour du village. Les informatrices les plus âgées parlent également de cette pratique, ce qui montre qu'elle est connue de longue date. En 1991, une peperuda a eu lieu dans le village pomak de Kirkovo où un prêtre chrétien a participé à la procession. Aussitôt après, il s'est mis à pleuvoir « - dit l'informatrice (ACE,1991). (Chez les Turcs l'essentiel du rite consiste dans le ramassage de "pierres noires" - au nombre de 900, de 9000, de 99000 - que les participants doivent jeter dans la rivière, après la prière du hodja). A Ardino, on a fait le mevlid le même été de 1991. Après quelques heures, il s'est mis à pleuvoir - affirment les informateurs (ACE, 1991).

\section{Le cycle de vie et les rites familiaux}

Des parallèles entre les fêtes et les rites des différentes communautés ethniques et confessionnelles existent dans les célébrations des étapes essentielles du cycle de la vie. Cette catégorie de rites, tout comme les rites saisonniers sont enrichis par des pratiques non canoniques.

C'est le cas notamment du rite surnommé Pita (pain rituel) chez les chrétiens et Bebelik chez les musulmans, qui intervient le quarantième jour après la naissance et qui a comme objectif l'adoption du nouveau-né par les femmes de la famille.

Bien que les rites funéraires, très différents pour chacune des communautés confessionnelles, respectent scrupuleusement les prescriptions de leur canon religieux respectif, ils dénotent également des éléments païens communs ou similaires. Et on ne manque pas d'inviter à un enterrement des représentants de l'autre communauté confessionnelle.

L'inauguration des travaux de construction d'une nouvelle maison exige un rite sacrificiel commun à toutes les communautés confessionnelles et ethniques. Il s'agit de l'immolation d'un animal (kurban) au-dessus des fondements du nouvel édifice. L'achèvement des travaux et l'investiture du lieu exigent également un kurban.

Dans le contexte urbain, le double rite sacrificiel, bien que simplifié et modifié, est également observé par tous. Le premier kurban intervient lors de l'installation de la famille dans un nouvel appartement. Ainsi le soir même de ce jour inaugural, on immole un coq et on invite au festin familial les plus proches parents. Environ un an plus tard, l'installation dans le nouvel habitat est célébrée par un grand kurban et une consécration religieuse solennelle auxquels on invite beaucoup de monde (ACE, 1991). Ce rite donne de l'assurance aux générations transitoires qui réalisent la rupture pénible avec l'espace et la vie communautaire de la société traditionnelle. Ainsi par exemple, nous avons vu dans 
le salon d'une jeune famille turque du « complexe » Vazrozdenci à Kardzali, un balai orné d'un ruban rose, évidemment un cadeau de noce. Dépourvu de son ancien usage fonctionnel, il assumait donc sa nouvelle fonction purement symbolique de signe assurant la protection du jeune couple.

Tous ces rites relatifs à la construction et l'installation dans une nouvelle maison participent de comportements religieux populaires.

\section{Croyances}

Selon une grande partie de la population rhodopienne, y compris celle des villes, le monde est peuplé d'esprits et génies invisibles, bons et mauvais, que l'on doit respecter et honorer, afin de s'assurer leur protection contre le malheur. Aussi, se livre-t-on à toute sorte de pratiques magiques (ACE, 1991). Ce ne sont pas tant les croyances et les rites euxmêmes qui témoignent de l'influence mutuelle et de l'interpénétration des cultures que la désignation des puissances surnaturelles par des noms étrangers à la langue pratiquée par le groupe ethnique concerné. Par exemple une puissance surnaturelle malfaisante sera désignée par les chrétiens comme par les musulmans par les noms de dracus (gr.), de vrag (bulg,) ou de shejtan (arabe).

39 La croyance en l'existence d'une série de puissances maléfiques assez semblables pour tous les groupements ethniques a une portée générale. Ces esprits malfaisants font souffrir les hommes en provoquant toutes sortes de malheurs et maladies et parfois mortels. Ils sont censés habiter des endroits fixes, connus de tous : des cimetières, des maisons abandonnées, des terrains dangereux, des formations rupestres, nombreuses dans la région, parfois taillées par les hommes. Une partie de ces pierres, petites ou grandes, sont tenues pour sacrées et on n'ose pas les toucher (ACE, 1991).

Les croyances liées au serpent sont très répandues. Protecteur de la maison, il est désigné comme stopan, c'est-à-dire "propriétaire » ou "maître des lieux » par les Chrétiens et comme saybiya (même sens) par les Musulmans. Dans certains villages pomak le nom de stopan est attribué à un esprit maléfique.

41 La croyance au pouvoir des méchantes sorcières (mamnici) est très vivante chez les femmes, toutes confessions confondues. Sans les nommer explicitement, elles affirment que le village est toujours habité par des mamnici.

42 Pour se protéger contre le «mauvais oeil » (uroki), tout le monde peut se confier aux femmes exorcistes. Les seules personnes de sexe masculin qui peuvent être investies des pouvoirs sorciers ou magiques sont les prêtres musulmans. C'est pourquoi, quand il s'agit d'avoir recours à la magie curative, préventive ou protectrice, musulmans et chrétiens n'hésitent pas à s'adresser aux hodja.

Dans toute la région, les différents groupes de populations croient fermement au pouvoir sacré guérisseur de sources, de roches naturelles ou taillées de forme particulière, de certains endroits. Aux grands arbres alentour sont accrochés en offrande différents vêtements, serviettes et tissus. Nous avons visité plusieurs lieux sacrés vénérés par un groupe d'agglomérations villageoises ou citadines, ou bien par toute la population de la région, tels que le lieu sacré près du village de Kirkovo et le « Pont au diable » (Djavolski most), situé près du village d'Ardino. Ils sont connus pour leur pouvoir curatif et si l'on en jugeait par la quantité de vêtements déposés, toute la population devrait croire à leur influence bénéfique (ACE, 1991). 

tas) a été déformée mais n'a pas perdu sa fonction culturelle.

\section{Les controverses intraconfessionnelles musulmanes}

La totalité des institutions rituelles des musulmans rhodopiens dénote l'influence de l'alévisme qui n'est pas considéré comme un écart de l'Islam sunnite. Le témoignage en est l'interprétation de la fête Asura qui marque pour les shiite le jour de la mort de Hussein, fils d'Ali et petit-fils de Mahomet, le héros-victime des Alevi. Pour les Musulmans de la région, Asura serait plutôt un jour dédié aux morts rappelant quelque peu les zadusnici (fêtes-des-âmes-mortes) chrétiennes (ACE, 1991).

\section{L'équilibre fragile}

$\mathrm{Au}$ terme de cette brève analyse des institutions rituelles rhodopiennes on constate qu'au-delà des différences, certes, pertinentes, entre les pratiques religieuses chrétiennes et musulmanes, se dessine un espace commun où se mêlent des us et coutumes, ainsi qu'une série de croyances similaires ou même identiques qui relèvent d'une authentique religiosité populaire soutenue et argumentée par les règles de vie communautaires d'une société rurale.

Il ne faut pas cependant surestimer la fonction conciliatrice du système rituel. En effet, même si à certains égards l'habitus des rhodopiens apparaît comme un modèle de coexistence pacifique, rappelons-nous que la région fut, et elle reste encore d'une manière latente le foyer de l'intolérance ethnique. Il ne faut pas oublier que les plaies des abus du régime totalitaire sont toutes fraîches et surtout que les champs de batailles de Sarajevo ne sont pas loin d'ici.

\section{BIBLIOGRAPHIE}

Archives du Centre d'Ethnologie (ACE). LeRhodope oriental, 1991.

Christakudis, A., 1992, « Ikonomikata i pravata na mjusjulmanite v Garcija » (L'économie et les droits des Musulmans en Grèce). in : Aspecti na etnoKulturnata situacya v Balgarya, pp. 51-58.

Dimitrov, S., 1988, « Sastojanie i zadaci na demografskite proucvanija v Iztocnite Rodopi » (L'état et les objectifs des recherches démographiques dans la région du Rhodope oriental). in : Demografski i etnosocialni problemi v Iztocnite Rodopi (Problèmes démographiques et ethnosociologiques dans le Rhodope oriental), Plovdiv, pp. 5-41.

Gueorguieva, T., 1992, « Compatibilités et divergences dans les rapports quotidiens des Chrétiens et Musulmans en Bulgarie ", in : Aspekti na etnokulturnata situacya v, Balgarya, Materyali ot seminar, organiziran ot Centar za demokraciiia (Aspects de la situation ethnoculturelle en Bulgarie, Matériaux 
du Séminaire organisé par le Centre de la démocratie), Sofia, Fondation « Friedrich Neumann », pp. 138-151.

Konstantinov, J., Gulbrand, A.A. et Birgit, I., 1991, « Names of the Bulgarian Pomaks », in : Tromso University Working Papers of Language and Linguistics, Nordlyd, n 27, pp. 23-29.

Petkov, K. et Fotev, G., (éd.), 1990, Etniceskyat konflikt v Balgarya

Petkov, K. et Fotev, G., (éd.),1989, (Le conflit ethnique en Bulgarie en 1989). Sofia, 1990, Sociologiceski ar XIV.

Popov, V., 1992, « Ciganite v Balgarija i texnoto etnicesko samosaznanie » (Les Tsiganes et leur conscience ethnique identitaire). in : Aspekti ... , pp. 118-124,

Stoilov, K., 1992, « Sotvoreni vrati kam sveta i xorata » (Les portes ouvertes vers le monde), in : Aspekti..., pp. 169-174.

Popova, A., 1985, « Dvata patja na slanceto » (Les deux chemins du soleil), in : Vekove, Sofia, $\mathrm{n}^{\circ} 5$, pp. 5-15.

Popova, A., 1990, « Les fils du temps », in : Civilisations - Ethnologie d’Europe, n 3 (Les Plantes et les Saisons, calendriers et représentations ), pp. 23-39.

\section{NOTES}

1. L'article qui suit fait état des premiers résultats de deux séries d'enquêtes réalisées en été et automne de l'année 1991 dans le Rhodope Oriental de Bulgarie. Le programme de recherche, dirigé par le prof. Tsvetana Gueorguiéva, a été élaboré par le Centre d'Ethnologie de la Faculté d'Histoire de l'Université de Sofia «St. Clément d'Ohrid».

2. Une partie des résultats ont été publiés dans l'article de Tsvetana Guéorguiéva, 1992.

3. On désigne par ce terme de «complexe» un ensemble de constructions style HLM qui forment un quartier moderne.

4. Les recherches centrées sur la culture traditionnelle de la population du Rhodope oriental sont nombreuses, Toutefois, dans leur grande majorité, elles soulignent son caractère prétendument monolithique, d'essence bulgare, A titre d'exemple on pourrait citer l'article de Dimitrov, 1988.

5. Ce paragraphe concernant l'usage combiné de différents calendriers, ainsi que la systématisation du registre pluriel des institutions rituelles (cf. infra) est élaboré en collaboration avec A. Popova. Sur la problématique calendaire voir également Popova, 1985 et 1990.

6. Le calendrier civil en réalité fait partie intégrante du calendrier religieux chrétien, Et si nous lui avons réservé une place autonome dans le classement. c'est en raison de son statut exclusif à l'époque du régime totalitaire hostile à toute manifestation religieuse, qu'elle soit chrétienne, musulmane ou autre, Même l'Eglise orthodoxe n'était pas autorisée à vendre ou à distribuer librement le calendrier religieux de l'année en cours, Et si la liberté confessionnelle était reconnue officiellement, aucun citoyen bulgare sous aucun prétexte n'était pas autorisé à chômer le jour de Noël, sauf si celui-ci tombait le Dimanche, 11 en était de même pour toutes les fêtes religieuses, Quelques-unes des fêtes orthodoxes, en raison de leur grande popularité avaient reçu droit de cité soit sous une forme laïcisée, soit comme fêtes traditionnelles à caractère païen préchrétien, Ainsi la Saint-Georges devint «La fête du pasteur », la Saint-Tryphon - "La fête du vigneron », les Pàques - "La fête des oeufs peints », Mais bien entendu cette grâce ne touchait jamais aucune fête islamique, Cela n'empêchait pas les gens de célébrer en privé leurs fêtes religieuses. 
7. Le terme kurban, d'origine biblique (hébraïque). désigne aussi bien le rite de sacrifice sanglant que l'animal sacrifié, Rejeté en tant que pratique païenne de la liturgie chrétienne, dans les traditions populaires balkaniques le kurban a sauvegardé son caractère sacramentel. C'est ainsi que l'Eglise orthodoxe fut amenée à l'admettre : aucun kurban n'a lieu sans la bénédiction d'un pope.

8. Le nom bulgare de la fête est Velikden, « le Grand-jour ».

\section{RÉSUMÉS}

The fragile equilibrum of a harmonious coexistence of minoritary ethnic groups (Pomaks, Sunnite-Turcs, Schiite-Turcss and Tzigans) with the majoritary Bulgarian ethnie group of Bulgaria's South-East mountainous region relies in great part on the converging aspects of the respective ritual institutions. Indeed, the ritual practices of these ethnie groups of ruarl origin, besides confessionnal and cultural differences, are part of a common sphere determined by the mode of apprehension of a similar world as well as by a holistic rural society's rules of life.

\section{INDEX}

Mots-clés : ethnicité, religion, identité, cohabitation, Bulgarie

Keywords : ethnicity, identity, Bulgaria

\section{AUTEUR}

\section{GUEORGUIEVA TSVETANA}

Université de Sofia 